\title{
Delivery practices, hygiene, birth attendance and neonatal infections in Karamoja, Uganda: a community-based study.
}

\author{
Leah J Hopp
}

127 Wakefield Crescent, London, Ontario, N5X 1Z6, Canada

\begin{abstract}
Background: Drawing attention to home birth conditions and subsequent neonatal infections is a key starting point to reducing neonatal morbidity which are a main cause of mortality in sub-Saharan Africa.

Objectives: To determine the proportion of respiratory, ophthalmic, and diarrhoeal infections in neonates; the proportion of mothers of neonates, following clean delivery practices; and to explore existing community practices during delivery and the neonatal period.

Methods: A descriptive, cross-sectional, exploratory study, including 10 questionnaires and five Key-Informant interviews, in rural Karamoja, Uganda.

Results: Post-delivery razor blade and string use was $90 \%$, but clean delivery surface use only $30 \%$, while $90 \%$ obtained bathing water for neonates from boreholes. No mothers washed hands after latrine-related activities compared with $83 \%$ for food-related activities. None delivered in health centres or with skilled birth attendants. Respiratory infections occurred in eight neonates, compared to two ophthalmic infections, and no diarrhoea.

Conclusion: Use of clean delivery surfaces needs to be improved as well as washing after latrine-related activities. Diarrhoea was far less common than expected. Since rural Mother-Infant pairs spend the majority of their post-delivery time around the homestead, hygiene impacts neonatal infections to a large degree, possibly even more so than delivery practices.

Keywords: Neonatal, birth, delivery, clean delivery kit (CDK), clean delivery practices, infection, diarrhoea, ophthalmic, respiratory, hygiene, traditional birth attendant (TBA), home, rural, community, Nakaale, Nakapiripirit, Karamoja, Uganda.
\end{abstract}

DOI: https://dx.doi.org/10.4314/ahs.v17i1.3

Cite as: Hopp LJ. Delivery practices, bygiene, birth attendance and neonatal infections in Karamoja, Uganda: a community-based study. Afri Health Sci. 2017;17(1): 7-13. https:/ / dx.doi.org/10.4314/ abs.v17i1.3

\section{Introduction}

Of the 3.3 million annual neonatal deaths, sepsis accounts for $15 \%{ }^{1}$. Approximately $99 \%$ of neonatal deaths take place in developing countries, mostly in homes and communities ${ }^{2}$. Transmission of neonatal sepsis during delivery may lead to $30-40 \%$ of infections ${ }^{1}$. Infection causes $36 \%$ of neonatal deaths in low-income countries ${ }^{3}$. Deaths due to infectious causes (64\%) were $40 \%$ likely to occur in the neonatal period, contributing to 7.6 million deaths of children less than five years old ${ }^{4}$. While infection rates
Corresponding author:
Leah J. Hopp,
127 Wakefield Crescent,
London, Ontario, N5X 1Z6, Canada
+15194551220
P.O. Box 1307, Mbale, Uganda
Tel: +256781410055
Email: leahhopp@gmail.com

are already known for children under five years from the local HC II and regionally, there is no specific data for the neonatal period. Matany hospital states that $90 \%$ prefer to deliver unassisted ${ }^{5}$. Neonatal care is not available to many in the rural areas of developing countries because post-natal care is non-existent ${ }^{6}$.

Despite a widespread understanding of the importance of cleanliness as a means to preventing neonatal infections, it does not always reflect adequate implementation of clean delivery practices, especially in community settings ${ }^{9}$. Clean delivery practices and neonatal hygiene could prevent many needless deaths, specifically where the majority of births still take place at home.

This study's objectives for Nakaale parish were: first, to determine the proportion of neonatal respiratory, ophthalmic, and diarrhoeal infections; second, to determine the proportion of mothers of neonates, following clean delivery practices; third, to explore existing community 
practices during delivery and the neonatal period.

\section{Methods}

A mixed research method using an exploratory strategy was used. Quantitative data from questionnaires was collected from 10 Karimojong mothers of neonates, while qualitative data was collected from five Key-Informant (KI) interviews. Data was collected from villages in $\mathrm{Na}$ kaale parish, Nakapiripirit district, Karamoja, Uganda. Data was collected from July 22-August 22, 2013. Only $33 \%$ of births in Uganda are registered, while in Karamoja, only $8 \%$ have a birth certificate ${ }^{10}$. Because a birth registry was not reliable for Nakaale parish, and because 63\% are born outside of a $\mathrm{HC}^{10}$, random sampling could not be used. Purposive sampling was employed by having local Karimojong inform the Principle Investigator (PI) of women from their sub-village with neonates. Informants freely revealed news of all their neighbour's births to the PI with no selection bias. The PI also visited villages asking about women who were pregnant in order to note their expected date of delivery so a follow-up visit could include them in the study. New mothers were questioned at home. Immunization records were not used to locate women with neonates because women living in Nakaale parish seek health care from five different surrounding HCs.

KI's were chosen in a purposive way based on their knowledge of local customs surrounding delivery or their leadership in the community. During KI interview selection, the saturation point was considered to have been reached when no new information was acquired. At the point of saturation, five KI's had been interviewed: a Traditional Birth Attendant (TBA), a clinic health worker, a male community leader, a female community leader, and an elderly woman.

The questionnaire and interview guide were conceptualized in English before being pre-translated into Ngakarimojong. The closed, coded questionnaire was verbally conducted in Ngakarimojong. KI interviews, implementing a semi-structured guide, were conducted in English with simultaneous translation into Ngakarimojong.

Interview data completeness was augmented through written notes and audio recording which were also transcribed. Data validity was ensured by pre-testing for language clarity, translation issues, and length acceptability.
Data quality was improved through personal collection by PI, together with bi-lingual language experts. Incomplete and missing data was identified and resolved on the same day as data collection and entered into a spreadsheet, rather than after the study's completion to increase accuracy and consistency.

The main dependent variable was infection status (measured as the proportion of neonates with respiratory infection, ophthalmic infection and/or diarrhoea). Independent variables included: demographic characteristics, delivery practices, hygiene practices, and birth attendance; and were hypothesized risk factors for infection. Analysis was performed using STATA $11.1^{11}$.

Manifest content analysis was performed on the data obtained from the KI interviews. Condensation, aggregation and abstraction were used to create categories, sub-categories, and codes regarding transmission of infection, birth preparedness, and unassisted deliver $y^{12}$.

Before questioning, each mother consented, with a fingerprint, in the presence of a witness. The written consent form was verbalized to each participant due to functional illiteracy.

\section{Results}

Ten women with neonates were questioned with their ages ranging from 18-45 years. The women's ages were estimated based on the guessed age of their eldest child because dates of birth are not remembered or recorded in rural Karamoja. The exact neonatal ages in the study were measured accurately. The mean neonatal age was 11 days $(+/-1 \mathrm{SD}=6.6 ; \mathrm{CI}=5.3,15.7)$ and the median was 10 days $(\mathrm{IQR}=9)$. While formal education is obtained through official scholastic channels, "informal learning is never organised, has no set objectives in terms of learning outcomes and is never intentional from the learner's standpoint. Often it is referred to as learning by experience" 13 .

Eight neonates had respiratory infections; while only two had ophthalmic infections and none had diarrhoea. No treatment was sought for any of the infections. Respiratory infections were also present among family and neighbours in $63 \%$ of the cases, while ophthalmic infections were not. In four neonatal respiratory cases, the mother also had a respiratory infection. The median duration for respiratory infections was 4.5 days $(\mathrm{IQR}=5.5)$ and the 


\begin{tabular}{|l|l|l|}
\hline \multicolumn{2}{|c|}{ Table 1: Demographic Characteristics of Mother-Infant Pairs in Rural Karamoja, 2013} \\
\hline Variable & Category & N=10 \\
\hline Age of Neonate (in days) & $\leq 7$ & 4 \\
\hline & $>7$ & 6 \\
\hline Birth Order of Child & $\leq 2$ & 3 \\
\hline & $>2$ & 7 \\
\hline Antenatal Care (ANC) visits & $<4$ & 2 \\
\hline & $\geq 4$ & 8 \\
\hline Existence of a Co-wife & No & 6 \\
\hline & Yes & 4 \\
\hline Sharing a Hut (number of people) & $\leq 4$ & 8 \\
\hline & $>4$ & 2 \\
\hline Mother's Education & Informal & 8 \\
\hline & Formal & 2 \\
\hline Male Partner's Education & Informal & 8 \\
\hline & Formal & 2 \\
\hline Mother's Job & Non-Remuneration & 10 \\
\hline & Remuneration & 0 \\
\hline Male Partner's Job & Non-Remuneration & 7 \\
\hline & Remuneration & 3 \\
\hline & & \\
\hline
\end{tabular}

mean duration was six days.

The duration of sickness for all ophthalmic infections was three days.

Among neonatal clinical respiratory infections, the most common symptom $(80 \%)$ was a cough. All neonates with clinical eye infections also had itchy eyes, as declared by the mother. While the respiratory infections from the study followed what was expected for the Karamoja region, there were zero cases of diarrhoea in this study compared to the Uganda Demographic Health Survey

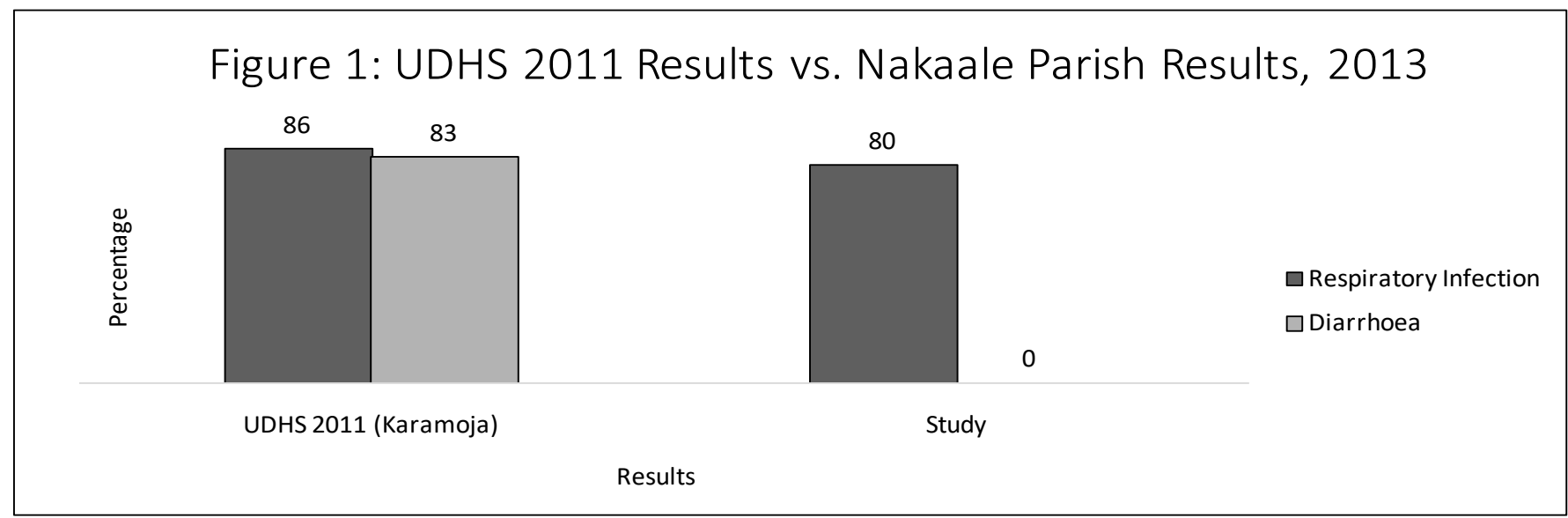

Figure 1: Respiratory infections were comparable among the UDHS 2011, Karamoja Region, results in children under five years old (86\%) \& Nakaale Parish study area (2013) results (80\%). Cases of diarrhoea in the UDHS 2011, Karamoja, (93\%) were high compared to cases from Nakaale (0\%). 
(UDHS) 2011 results $^{10}$ of 93\% for Karamoja (Figure 1). Unskilled birth attendance at delivery occurred in all of the respondents compared to a regional average of $59 \%{ }^{10}$. None of the respondents delivered in a HC. Only one mother used a Clean Delivery Kit (CDK), while others used only partial CDKs (Figure 2). Deliveries made on unsanitary birth surfaces $(n=7)$ included primarily the dirt floor of the hut, along with two respondents who used

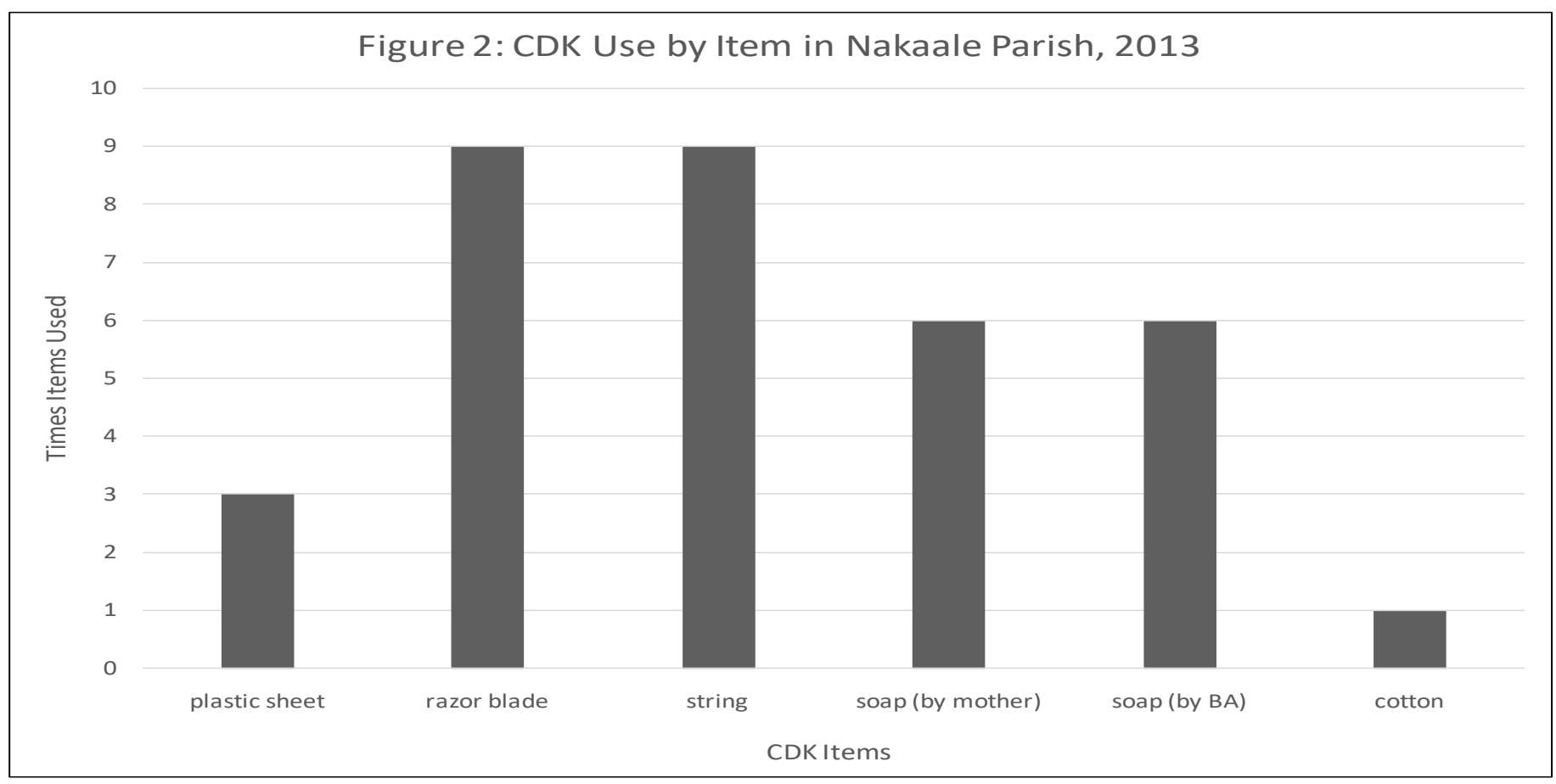

Figure 2: Clean Delivery Kit (CDK) items were used to various degrees by women on the day of delivery ( $\mathrm{N}=10)$. Plastic sheets (3), razor blades (9), string (9), soap - by the mother (6), soap - by the Birth Attendant (BA), and cotton (1).

either a grain sac or tarp.

None of the respondents reported giving colostrum during the first hour after delivery. Only two neonates in the study were without infection; however, none of the neonates in the study had all three infections: respiratory, ophthalmic, or diarrhoeal. None of the mothers delivered at a $\mathrm{HC}$ with a Skilled Birth Attendant (SBA). Two women delivered completely alone without even family or a neighbour to assist them. TBAs assisted in eight home births. Because all the deliveries were without SBA's, relationships between other variables could not be measured.

Neonatal bathing during the first six hours after delivery was reported by half of the mothers. Six mothers reported washing the neonate's face during this same period. Three mothers reported neonatal bathing and face-washing thrice daily. One Mother-Infant pair had not yet bathed after delivery because of a local tradition of waiting until the umbilical cord stump falls off, but all the others bathed with water from a borehole.

Washing of the hands by mothers was broken down into three main categories: latrine-related (including after defecation and after the neonate defecates), food-related (including before or after eating or cooking) and other (including "in the morning", "after work", and "before bathing the child"). Only one mother reported washing her hands after defecation, while six reported washing their hands specifically pertaining to eating or cooking. When mothers washed their hands after latrine-related activities, their neonates did not exhibit infections. When mothers washed their hands in food-related activities, some neonatal infections were present, but they were more likely to have only one of the three infections instead of two or more. The "other" category for hand-washing was not seen as significant.

Through interview, it was possible to learn more about what the community thought regarding transmission of infections. One KI shared about how a sheep needs to be killed in order for the neonate's infection to be healed because "if you take the child to the clinic when the local traditions have not been done, the child will not get well from the clinic" (KI-2). Another reason why it is thought that neonates 
become sick is because of malnourishment: " bunger can come to the child and it turns to sickness... When you are eating many times in a day, then even when you become sick, you can recover quickly" (KI-3) Three KI's said that God is the one who makes neonates sick. Following delivery, a woman does not live with her male partner, which is thought to prevent sickness: "When you see that the child is still young and you sleep with your husband, in Karamoja, it is not wanted. It is better when you deliver that you stay for four months, or five or also six" (KI-1). The neonate is kept inside the hut until after the umbilical cord stump falls off. This traditional practice helps to protect the cord stump from infections being brought by the wind: "Wind is bad. It can enter the cord and it can swell' (KI-3).

Birth preparedness is restricted by local tradition that forbids women to buy delivery supplies before the baby is delivered: "In Karimojong culture, if you buy a sheet and say that when I deliver I will use it to carry my child, then the child will die" (KI-1). Some items can be bought before delivery if they are also used for others: "You can even buy a basin for yourself for bathing, later it will be for the child" (KI-4).

During unassisted deliveries, some do not want or seek help, while others believe that the local traditions restrict them from seeking help during delivery. Younger women, with fewer deliveries, are more likely to seek assistance: "When a woman is old enough she can deliver alone" (KI-2). Some say that it is the choice of each woman whether she is able to deliver alone or not; however, others say that if the local traditions are not followed, then the delivery may be delayed: "You will not deliver that day. You will take like a full week in labour pain" (KI-1). If a woman delivers alone the first time, or at least one time, she is very likely to give birth alone during a subsequent pregnancy, according to one interviewee. Other interviewees explained that women are allowed to call for help during delivery and to have multiple assistants. Delivery assistance by an unskilled birth companion may be worse than delivering alone because people place their trust in TBAs that do not know danger signs or birth complications: "The bad thing at home is that, when it is not yet time for the baby to come, they tell you to push" (KI-1).

\section{Discussion}

The study area was limited because of rainy season inaccessibility. The sample size was small due to externally-imposed, data-collection time limits. Lack of a birth registry severely limited statistical analysis. Thus, conclusions were insignificant, instead tending towards possible explanations needing further study.

No treatment was sought for any of the study infections with zero transmission of ophthalmic infections and 63\% of respiratory infections. When clinical cases do not seek treatment, there is a greater likelihood that infections will spread. High prevalence of respiratory infections $(8 / 10)$ in the study reflects that of the community. This could be due to none of the neonates receiving colostrum within the first hour after birth. A possible source of ophthalmic infections could be from delivery on an unsanitary birth surface, such as the dirt floor of a hut. Other sources of ophthalmic infections could be via the birth canal, through the wind, or from greeting and touching the eyes without washing hands frequently. There were no cases of diarrhoea possibly because of the abundance of boreholes in the area. All mother-infant pairs lived closer to the borehole than to the river so it was believed that they used borehole water for most household activities. Respiratory infections were similar between this study and the UDHS 2011 Karamoja results for children under five years old ${ }^{10}$, but results for diarrhoea were not similar $(0 \%$ vs $93 \%$ ) (Figure 1) throughout their reproductive years. Some clinics in neighbouring parishes give incentives to those coming for TT immunizations. Receiving TT immunizations does not necessarily mean that women are receiving a full ANC checkup. The Doctor that has been managing AYPC HCII since 2009 reported zero umbilical cord infections. Dry cord care is commonly practiced in the region.

One area with room for improvement is the delivery surface, although post-delivery razor blade and thread use was high. If women endeavour to not deliver on an unsanitary surface $(7 / 10)$, then hopefully fewer infections will occur. Another area of improvement is washing hands with soap during delivery and immediately after by both the mother and birth companion, which was only practiced at six deliveries. CDKs used to be distributed during the fourth ANC visit at AYPC HCII, but now they are given as part of delivery fees. Because the sample size was very small there is no clear association between CDK items and unclean items.

When six delivery practices as a sub-set were amalgamated and compared with neonatal infections, the results were interesting. CDK use, when neonates were given 
colostrum, whether the mother and/or birth attendant used soap to wash their hands, birth attendance and birth place were decided to either be satisfactorily or unsatisfactorily practised. If a delivery practice was satisfactorily performed, then two points were given, and if it was unsatisfactorily performed, then one point was given. Each mother-infant pair scored between $6-12$ points. None of the mother-infant pairs scored $100 \%$ satisfactory on all six delivery practices and 60\% scored unsatisfactory on all practices. Among these mother-infant pairs, 33\% had both respiratory and ophthalmic infections, 33\% had no infection, and none had diarrhoea. Of the $40 \%$ that scored higher than $60 \%$ satisfactory, all had respiratory infections and none had either ophthalmic infections or diarrhoea.

It was expected that more than two women in the study would deliver alone based on anecdotal evidence. One of the only times that Karimojong women are praised by their husbands is when they are strong. When a woman delivers alone, without assistance, she is praised.

A Karimojong tradition, which is no longer widely practiced, but was found among one participant in the study, regards the first days after delivery. Until the umbilical cord stump falls off, the mother and neonate are not allowed to bathe or leave the hut; during this time, the woman is also forbidden to eat and drink. This can have detrimental neonatal effects, particularly when colostrum is delayed in being let down. Because the post-natal period is a crucial time for identifying infections and seeking care, isolation of mother and neonate from the community is an especially risky tradition ${ }^{14}$. Mothers may have reported what the PI wanted to hear, particularly regarding bathing frequency, in which case the actual practice is expected to be less frequent.

Use of clean water was higher in the study than in Karamoja possibly because a development initiative has drilled boreholes for many villages in the parish, increasing access to potable water for hygiene, as well as consumption. Because the homes were located closer to the boreholes than the river, most women used clean water for washing and cooking. It was surprising that more mothers reported washing their hands before food preparation than any other activity. Washing hands after defecation or helping a neonate was less often reported than ideal. While washing hands before preparing food and before eating food is very important, washing hands after defecation needs to be improved upon.

The following hygiene practices were analyzed together and compared with neonatal infections: When the neonate was first bathed, when the neonate's face was first washed, how many times it was bathed per day, how many times its face was washed per day, what water source was used for washing, and when the mother washed her hands. If a hygiene practice was satisfactorily performed, then two points were given, and if it was unsatisfactorily performed, then one point was given. Each mother-infant pair scored between 6-12 points. None of the mother-infant pairs scored $100 \%$ satisfactory on all six hygiene practices, but six of the mother-infant pairs scored $90 \%$ satisfactory. Of these mother-infant pairs, $22 \%$ had both respiratory and ophthalmic infections, $22 \%$ had no infections, and none had diarrhoea. Of the four that scored less than $90 \%$ satisfactory, all had respiratory infections and none had either ophthalmic infections or diarrhoea.

\section{Conclusion}

There was frequent use of post-delivery razor blades and threads, but improvement is needed for clean delivery surfaces. There was an acceptable amount of washing hands in food-related activities, but not after defecation. No women delivered with a SBA. Respiratory infections were common, but there were no cases of diarrhoea.

It is recommended that behaviour change communication (BCC) materials be developed regarding sanitary birth surfaces, alongside teaching about the benefits of initiating breastfeeding immediately after delivery, and the use of culturally specific BCC materials on washing of hands after defecation.

The following further research is recommended: repeat study in a more systematic way using a multi-disciplinary approach with more participants and over a longer period of time thus enabling better statistical analysis, such as logistic regression; conducting a qualitative study, with focus groups, to gather more in-depth knowledge about the reasons behind the behaviour and practices described in this study; and a study on the prevalence of diarrhoea in areas with adequate borehole coverage compared to areas without.

\section{Conflict of interest disclosure}

I, the author, declare that potential conflict of interest does not exist in this manuscript.

\section{Acknowledgements}

African Health Sciences Vol 17 Issue 1, March, 2017 
First of all, I praise God, the Father of my Lord Jesus Christ. Second, I thank Agnes Kiragga, for her valuable guidance throughout the analysis of this project. Third, I would like to thank the Orthodox Presbyterian Uganda Mission (OPUM) for allowing me to combine this project with my work. I could not have done research without the help of four very important people. Thank you to Olivia Naduk, Rose Acia, Paul Lokwii, and David Lokwii for their assistance with interpretation and communication in Ngakarimojong.

\section{References}

1. Seward N, Osrin D, Li L, Costello A, Pulkki-Brännström AM, Houweling TA, et al. Association between clean delivery kit use, clean delivery practices, and neonatal survival: pooled analysis of data from three sites in South Asia. PLOS Medicine. 2012;9(2), PubMed e1001180. DOI:10.1371/journal.pmed.1001180. (accessed 29 November 2012).

2. Bhutta ZA, Darmstadt GL, Hasan BS, Haws RA. Community-based interventions for improving perinatal and neonatal health outcomes in developing countries: a review of the evidence. Pediatrics. 2005;115(Supplement 2),519-617. DOI:10.1542/peds.2004-1441. (accessed 20 July 2013).

3. Lawn JE, Cousens S, Zupan J. 4 million neonatal deaths: When? Where? Why? The Lancet. 2005;365(9462), 891-900. PubMed DOI:10.1016/S0140-6736(05)71048-

5. (accessed 8 April 2013).

4. Liu L, Johnson HL, Cousens S, Perin J, Scott S, Lawn JE, et al. (2012). Global, regional, and national causes of child mortality: an updated systematic analysis for 2010 with time trends since 2000. The Lancet. 2012;379(9832),21512161. DOI:10.1016/S0140-6736(12)60560-1. (accessed 8 April 2013).

5. St. Kizito Hospital Matany. Moroto Diocese. Annual Analytical Report for the Financial Year 2011/12. 2012. Available from: matany.altervista.org/notiziari/ St_Kizito_Hospital_Matany_Annual_Report_2011-12. pdf. (accessed 12 July 2013).

6. Waiswa P, Kemigisa M, Kiguli J, Naikoba S, Pariyo GW, Peterson S. Acceptability of evidence-based neonatal care practices in rural Uganda - implications for pro- gramming. BMC Pregnancy and Childbirth. 2008;8(21). DOI:10.1186/1471-2393-8-21. (accessed 26 March 2014).

7. Blencowe H, Cousens S, Mullany LC, Lee AC, Kerber K, Wall S, et al. Clean birth and postnatal care practices to reduce neonatal deaths from sepsis and tetanus: a systematic review and Delphi estimation of mortality effect. BMC Public Health. 2011;11(Suppl.3),S11. DOI:10.1186/1471-2458-11-53-S11. (accessed 2 January 2013).

8. Yanagisawa S, Oum S, Wakai S. Determinants of skilled birth attendance in rural Cambodia. Tropical Medicine and International Health. 2006;11(2),238-251. DOI:10.1111/j.1365-3156.2005.01547.x. (accessed 20 July 2013).

9. Moyer CA, Aborigo RA, Logonia G, Affah G, Rominski S, Adongo PB, et al. Clean delivery practices in rural northern Ghana: a qualitative study of community and provider knowledge, attitudes, and beliefs. BMC Pregnancy and Childbirth. 2012;12:50. DOI:10.1186/1471-239312-50. (accessed 29 November 2012).

10. Uganda Bureau of Statistics (UBOS), ICF International Inc. Uganda Demographic and Health Survey 2011. Kampala, Uganda: UBOS and Calverton, Maryland: ICF International Inc. 2012. Retrieved from: www.measuredhs.com/pubs/pdf/FR264/FR264.pdf. (accessed 13 February 2013).

11. Stata/IC for Windows. [computer software]. Version 11.1. Texas (USA): StataCorp LP; 2009. Available from: www.stata.com

12. Graneheim UH, Lundman B.Qualitative content analysis in nursing research: concepts, procedures and measures to achieve trustworthiness. Nurse Education Today. 2004;24,105-112. DOI:10.1016/j.nedt.2003.10.001. ( PubMed accessed 26 March 2014).

13. Organisation for Economic Co-operation and Development. Skills Beyond School. (n.d.). Paris, France. Retrieved from: www.oecd.org/edu/skills-beyond-school/ recognition of non-formal and informal learning-home. htm. (accessed 12 June 2015).

14. Byaruhanga RN, Nsungwa-Sabiiti J, Kiguli J, Nsabagasani X. Hurdles and opportunities of newborn care in rural Uganda. Midwifery. 2010. DOI:10.1016/j. 